\title{
MARCHE ET COMMERCIALISATION DU RIZ LOCAL AU CENTRE-OUEST DE LA CÔTE D'IVOIRE
}

\author{
C. KOFFI ${ }^{1}$ et K. A. N'DRI ${ }^{2}$ \\ ${ }^{1}$ CNRA Station de recherche de Gagnoa \\ ${ }^{2}$ Université de Cocody, Abidjan
}

\begin{abstract}
RESUME
Face à une augmentation de la demande pour le riz et à une production nationale plus ou moins stable depuis 1980, la Côte d'Ivoire déploie beaucoup d'efforts dans la riziculture, notamment dans les bas-fonds. La présente étude vise à analyser l'efficacité des systèmes de commercialisation de riz local. La coexistence de trois grands circuits de commercialisation a été révélée : cycles court, moyen et long. Globalement, le marché est certes concurrentiel, mais on note des pratiques monopolistiques et oligopolistiques de certains intermédiaires commerciaux. Le prix du riz varie dans le temps et l'espace, et est dominé par les marges bénéficiaires. En outre, une proportionnalité existe entre les marges et les charges supportées par les différents opérateurs.
\end{abstract}

Mots-clés : Riz, commercialisation, circuit de distribution, intermédiaires, demande, Côte d'Ivoire

\section{ABSTRACT}

MARKETING OF DOMESTIC RICE IN CENTER-WEST CÔTED'IVOIRE

Faced with a growing demand for rice, and a national rice production, which has not increased since 1980 , Côte d'Ivoire is presently investing considerable effort in the development of rice culture, specially in inland valleys. The objective of the present study is to examine local marketing systems. The results show that there were three channels of distribution : (short, medium and long). The market was characterized by an unperfect concurrence, because of monopolistic and oligopolistic practices of some middlemen. The price of local rice was very variable in space and time. Margins and costs were quite proportional.

Key-words : Rice, outlet, marketing, Channel of distribution, middlemen, demand, Côte d'lvoire.

\section{INTRODUCTION}

Le marché ivoirien de riz est caractérisé par un déficit chronique. Malgré les efforts déployés par les pouvoirs publics, la production nationale de riz, soit 545000 tonnes de riz blanc en 2002, ne parvient pas à satisfaire la demande. l'Etat est donc contraint d'importer d'importantes quantités de riz, soit 350000 tonnes en moyenne par an depuis 1994, pour un coût total de 60 milliards de francs CFA selon Anonyme 1 (1997). Une des orientations de la politique de sécurité alimentaire menée en vue de résorber ce déficit autrement que par des importations massives, trop coûteuses en devises, est la promotion de la riziculture de bas-fonds. La région du Centre-Ouest réputée pour sa forte production de riz, soit $17 \%$ de la production nationale, est l'une des cibles principales de la politique d'intensification rizicole. Si des efforts sont faits pour augmenter la production, les systèmes de commercialisation font l'objet d'une attention moins forte depuis la faillite de l'exSODERIZ (Société pour le développement de la riziculture). De fait, les difficultés de commercialisation du riz local et d'approvisionnement en intrants et matériel divers comptent parmi les principales contraintes au développement de la riziculture. Aussi est-il impératif de mener des investigations sur les systèmes de commercialisation.

Le riz pourrait bien représenter un élément crucial de la politique de sécurité alimentaire si une production domestique pouvait être 
développée et écoulée à travers des systèmes de commercialisation efficients, capables de fournir une offre répondant aux exigences de compétitivité du marché international. De fait, la filière riz en Côte d'Ivoire a fait l'objet d'investigations scientifiques, mais elles traitent généralement de la production. Les études sur les systèmes de commercialisation sont rares et portent sur les marges bénéficiaires, les contraintes et les politiques agricoles.

Poelmans (1997) a trouvé un rapport de $11 \%$ entre la marge bénéficiaire et le prix de vente entre détaillants et consommateurs de riz local et importé à Bouaké, Centre de la Côte d'Ivoire. A Korhogo au Nord, ce rapport a été de $24 \%$. L'auteur a expliqué ces marges trop élevées, (la norme étant de 5 à $8 \%$ ), par l'insuffisance de concurrence entre les détaillants. Dahoun (1998) a noté que dans le Centre-Ouest de la Côted'Ivoire, le prix du riz de bas-fond n'est pas incitatif. II ne précise pas, néanmoins, si ce prix est rémunérateur pour les facteurs de production, ni le seuil au dessus duquel le prix serait incitatif. Selon Yao (1985), l'échec de l'ex-SODERIZ s'explique, en partie par les erreurs de politiques agricoles. II a indiqué que les prix étaient fixés de telle sorte que la filière ne pouvait s'équilibrer. De fait, aussi bien le producteur que le consommateur étaient subventionnés. Le prix au producteur était supérieur au prix d'équilibre du marché pour encourager la production en vertu de la thèse de l'industrie naissante. Quant au prix au consommateur, il était inférieur au prix d'équilibre du marché pour satisfaire les consommateurs urbains. Au total, des investigations plus approfondies sur les systèmes de commercialisation du riz local apparaissent nécessaire.

L'étude se propose d'identifier les systèmes de commercialisation et apprécier leur éfficacité. De manière spécifique, il s'agit d'analyser les systèmes de commercialisation ; d'étudier la structure du marché et du prix ; les variations du prix dans les temps et dans l'espace ; et de faire des recommandations de politique économique.

\section{MATERIEL ET METHODES}

\author{
MATERIEL
}

\section{Echantillonnage}

Une pré-enquête exhaustive a été faite pour identifier l'ensemble des zones de production et les opérateurs qui interviennent dans la filière dans la région de Gagnoa. Au terme de cette pré-enquête, qui a permis d'interviewer 200 riziculteurs et 150 intermédiaires commerciaux, un échantillon de producteurs et un autre d'intermédiaires commerciaux ont été collectés. L'échantillon de producteurs a été de 60 individus, dont 13 en milieu urbain (ville de Gagnoa), 27 en milieu périurbain, 20 en milieu rural. 45 intermédiaires commerciaux ont également été choisis en milieu urbain (ville de Gagnoa), dont 15 grossistes et transformateurs et 30 détaillants. Pour le choix des producteurs, un échantillonnage à deux degrés a été fait. Le premier degré a consisté à tirer de façon aléatoire 9 villages en milieu rural et péri urbain, et le second a également été fait de façon aléatoire à l'aide des paysans dans ces villages. Pour les intermédiaires commerciaux, nous avons procédé à un échantillonnage aléatoire pour sélectionner les opérateurs à enquêter a été réalisé.

\section{Collecte de données}

Pour une meilleure analyse, des données primaires ont été collectées en 2004 à travers des enquêtes à passage unique, pour les données qualitatives, et à passages répétés, à fréquence quotidienne pour les données quantitatives auprès des opérateurs de la filière (producteurs et commerçants). Ces données ont concerné pour l'essentiel la démographie (ethnie, âge, sexe, niveau d'instruction, profession, etc), les entrées et sorties (charges fixes et variables, production, vente, autoconsommation), les modes de financement et de règlement de transaction, les contraintes, les prix, les 
systèmes de production et les circuits de distribution, les actifs et passifs, à court, moyen et long terme. Les données primaires ont été au besoin, complétées par des données secondaires obtenues par des travaux antérieurs.

\section{Présentation de la zone d'étude}

Les caractéristiques géographiques, climatiques et pédologiques sont celles de la moyenne Côte d'Ivoire pré-forestière. Le climat est tropical humide avec une végétation de type forêt dense. Mais, la forêt primaire a été progressivement remplacée par une végétation secondaire dominée par les jachères à Chromolena odorata. On observe dans la région deux saisons de pluies : la première de avril à juillet, et la seconde de septembre à octobre. Le niveau moyen des précipitations varie entre $1400 \mathrm{~mm}$ et $1600 \mathrm{~mm}$. La durée de la saison culturale est supérieure à 9 mois.

L'agriculture de plantation est dominée par les allochtones venus des régions du Centre et du Nord de la Côte d'Ivoire et des allogènes venus des pays limitrophes (Burkina Faso et Mali) qui constitue la main-d'œuvre agricole salariée. La densité de la population est élevée, soit $103 \mathrm{hab} / \mathrm{km}^{2}$ à Sinfra, $98 \mathrm{hab} / \mathrm{km}^{2}$ à Daloa et 79 hab/km² à Gagnoa (Anonyme 2, 1998). Les systèmes de production agricole sont diversifiés. Les cultures d'exportation (café, cacao, banane) coexistent avec les cultures vivrières (maïs, igname, banane, riz, manioc, cultures maraîchères). Le riz est la denrée alimentaire la plus cultivée dans la région.

L'encadrement des paysans est assuré par l'Agence Nationale pour le Développement Rural (ANADER), à l'exception des pisciculteurs. Une réelle dynamique de développement piscicole existe aujourd'hui aussi bien en milieu péri-urbain qu'en milieu rural. Le système de pisciculture vulgarisé est de type artisanale intégrée aux systèmes agricoles. L'encadrement est actuellement assuré par l'Association Pisciculture et Développement Rural au CentreOuest de la Côte d'Ivoire (APDRACI) qui a pris la relève des activités piscicoles initiées dans la région depuis 1980 par le projet de développement de la pisciculture en milieu rural qui couvrait $65 \%$ du territoire national, puis divers micro-projets piscicoles régionaux à Daloa et à Gagnoa entre 1990 et 1994 (Koffi et al., 2005). Selon ces auteurs, de nombreux bas-fonds sont disponibles. En 1980, 10255 ha de bas-fonds ont été aménagés dans la zone forestière de l'Ouest et du Centre-Ouest. Les périmètres sous barrages représentent 2,6\% de cette superficie. Les prises au fil de l'eau sont les plus nombreuses $(97,4 \%)$.

\section{METHODES}

L'identification des circuits de distribution a été faite à partir d'une approche fonctionnelle (fonction de production, de manutention, de transport, de financement, etc) et institutionnelle (agents et intermédiaires du processus de commercialisation).

L'analyse des tendances et de la variation des prix a été faite à partir d'instruments statistiques courants (moyennes, écarts types, pourcentages)

Pour l'analyse de l'efficience, la méthode d'analyse des marges (structure du prix) et celle de la structure du marché (SCP) ont été combinées, car cela nous a paru plus pertinent. En effet, trois méthodes ont permis d'analyser la performance des marchés agricoles. II s'agit des méthodes d'analyse des marges, de structure-conduite-performance et d'intégration des marchés.

La méthode d'analyse des marges procède par décomposition du prix soit dans une approche statique " concurrent method» ou dynamique «time lag method». (Raju et Van Oppen, 1982). Elle présente l'avantage de donner une vue d'ensemble des coûts et marges de commercialisation de la filière.

La méthode SCP établit une relation de causalité entre la structure et la performance du marché (Perrault, 1984). Elle permet de déterminer l'efficacité de la commercialisation, car l'efficience découle du degré de perfection du marché. Le marché de concurrence pure et parfaite est par essence un marché efficient.

La méthode d'intégration des marchés a été utilisée par certains économistes qui considèrent qu'un marché bien intégré est performant (Raju et Van Oppen, 1982). L'intégration de deux marchés est le degré auquel la formation des prix sur un marché est influencée par les prix de l'autre. La mesure de l'intégration se fait par les coefficients de corrélation des prix. Cette méthode n'a pas été retenue, car dans l'interprétation des marges et des coefficients 
de corrélation, il y a indécision dans certaines situations selon Raju, et Van Oppen (1982). C'est le cas, par exemple, de deux marchés où une collusion des vendeurs a eu pour retirer de fortes marges. Dans ce cas, on observe une forte corrélation des prix et des marges élevées. La situation inverse est observée sur deux marchés dont le flux de produits change temporairement de direction. On observe à la fois des prix faiblement corrélés et des marges faibles.

\section{RESULTATS}

\section{CARACTERISTIQUES SOCIO-ECONOMIQUES DES OPERATEURS}

La dimension socio-économique comprend les éléments démographiques, l'expérience, le niveau d'instruction, la profession, et les motivations.

\section{Eléments démographiques}

Les opérateurs de la filière sont de diverses origines (Tableau 1): autochtones, allochtones et allogènes. Les allochtones venus du nord ont représenté $50 \%$ des offreurs, $80 \%$ des grossistes et $57 \%$ des détaillants. Les allogènes (Burkinabés et Maliens) ont représenté $20 \%$ des grossistes et $33 \%$ des détaillants. Quoique gros producteurs de riz pluvial, les autochtones n'ont représenté que $10 \%$ des offreurs, ce qui atteste de l'importance de l'autoconsommation. La filière du riz commercial a été dominée par les hommes avec $97 \%$ des offreurs, $100 \%$ des grossistes et $56 \%$ des détaillants. L'essentiel de l'offre provient de la riziculture de bas-fond. L'âge moyen a été de 46 ans pour les offreurs, 37 ans pour les grossistes et 32 ans pour les détaillants (Tableau 2).

\section{Niveau d'instruction et experience professionnelle}

Comme le révèle le tableau 3 , les offreurs et les détaillants ont été en majorité des illettrés ( $70 \%$ et $60 \%$ respectivement), alors que les grossistes sont lettrés pour la plupart. Presque tous ont une bonne expérience en production ou en commercialisation du riz local (Tableau 3 ).

Tableau 1 : Répartition des opérateurs de la filière selon l'origine.

Distribution of operators according to origin.

\begin{tabular}{lccc}
\hline & \multicolumn{3}{c}{ Pourcentage des opérateurs (\%) } \\
\cline { 2 - 4 } Origine & Producteurs ${ }^{2}$ & Grossistes & Détaillants \\
\hline Autochtones & 10 & 0 & 10 \\
Allochtones & 50 & 80 & 57 \\
Allogènes & 40 & 20 & 33 \\
Total & 100 & 100 & 100 \\
\hline Source : données de l'étude $;{ }^{2}$ II s'agit des riziculteurs de bas-fond. Les autochtones pratiquent surtout la \\
riziculture pluviale pour l'autoconsommation
\end{tabular}

Tableau 2 : Répartition des opérateurs de la filière selon l'âge, l'expérience et le sexe.

Distribution of operators according to age, experience and sex.

\begin{tabular}{lccc}
\hline & \multicolumn{3}{c}{ Nombre et pourcentage d'opérateurs } \\
\cline { 2 - 4 } Paramètres & Producteurs & Grossistes & Détaillants \\
\hline Age (ans) & $46(12)$ & $37(11)$ & $32(10)$ \\
Expérience (ans) & $11(8)$ & $7(4)$ & $5(4)$ \\
Hommes (\%) & 97 & 100 & 57 \\
\hline Source : données de l'étude ; Les chiffres entre parenthèses sont les écarts types
\end{tabular}


Tableau 3 : Répartition des opérateurs selon le niveau d'étude.

Distribution of operators according to level of education.

\begin{tabular}{lccc}
\hline & \multicolumn{3}{c}{ Pourcentage des opérateurs (\%) } \\
\cline { 2 - 4 } Niveau d'éducation & Producteurs & Grossistes & Détaillants \\
\hline Illettrés & 70 & 20 & 60 \\
Primaire & 20 & 54 & 30 \\
Secondaire & 10 & 13 & 10 \\
Supérieur & 0 & 13 & 0 \\
Total & 100 & 100 & 100 \\
\hline Source : données de l'étude & & &
\end{tabular}

\section{CONTRAINTES LIEES \\ ALACOMMERCIALISATION}

Les contraintes à la commercialisation du riz local sont liées au capital, à l'état défectueux des routes et à l'étroitesse du marché en milieu rural.

\section{Contrainte liée au capital}

L'autofinancement est la règle dans le secteur. Aussi le capital est-il limitant. Cela entraîne l'inaccessibilité des intrants agricoles et des équipements modernes de production à la majorité de ces petits paysans.

\section{Etat défectueux des routes}

Le mauvais état des pistes villageoises rend l'accès à certaines zones de production difficile voire impossible surtout en période pluvieuse. De fait, les moyens de transport de la production du champ au village sont rudimentaires (sur la tête, à vélo ou à moto). Pour écouler leurs produits sur le marché local ou sur les marchés des villes les plus proches, certains paysans utilisent les mêmes moyens de transport ce qui entraine un surenchérissement des coûts.

\section{Perspectives de débouchés}

Dans l'état actuel du marché, le problème de débouché en milieu urbain ne se pose pas. Dans certaines zones, en milieu rural, l'étroitesse du marché fait de la desserte des marchés urbains une exigence du développement de la riziculture.

\section{CYCLES DE COMMERCIALISATION}

Trois principaux cycles de commercialisation coexistent : les cycles long, moyen et court. Dans la plupart des cas, le cycle de commercialisation est long. Le riz part du producteur au consommateur en transitant par trois intermédiaires commerciaux (collecteur, grossistes -transformateurs et détaillants). Dans le cycle moyen, ce sont les détaillants qui, en l'absence de grossistes, assurent le décorticage du riz. En milieu rural où se déroule une part infime de l'activité commerciale, le cycle est court. Les producteurs décortiquent le paddy (le plus souvent à la main) et vendent directement aux consommateurs. La plupart des producteurs visent le marché urbain, où ils peuvent vendre des quantités plus importantes de riz.

\section{FONCTIONS COMMERCIALES}

On a enregistré 3 intermédiaires commerciaux dans la filière. Ce sont : le collecteur, le grossiste et le détaillant.

Le producteur vend souvent son produit à des collecteurs ou à des grossistes spécifiques si ceux-ci ont financé la production. Aussi, souvent les producteurs empruntent de l'argent à ces intermédiaires commerciaux pour faire face à des besoins pressants, par exemple, la scolarité des enfants, les frais médicaux, etc. Aussi, le producteur est-il contraint de leur vendre sa production par contrat signé par les deux parties. Certains paysans qui se sont autofinancés n'ont aucune contrainte vis à vis des tiers et vendent leur produit lorsque le prix proposé par l'acheteur leur paraît rémunérateur.

Le collecteur assure l'activité de collecte du produit sur les marchés hebdomadaires ruraux ou aux abords des champs, puis achemine le riz vers les grossistes ou les transformateurs situés en milieu urbain. Le collecteur travaille, soit pour son propre compte, soit pour le compte des grossistes ou des détaillants basés sur les marchés urbains.

Quant au grossiste, il est basé en milieu urbain. Ce dernier assure le décorticage du riz qu'il revend aux détaillants. II sert souvent de bailleur de fonds au producteur qui est alors obligé de lui vendre sa production. 
En ce qui concerne le détaillant, on le retrouve souvent sur le marché urbain (lieu physique). II tient aussi des boutiques et des tabliers dans les quartiers de la ville.

\section{MODE DE REGLEMENT DES TRANSACTIONS.}

Les paysans autofinancés (18\%) vendent leur produit au comptant, alors que ceux qui sont financés par les intermédiaires commerciaux (82\%) vendent souvent cash, mais parfois, par crédit fournisseur. II en est de même des transactions entre les intermédiaires commerciaux. Seuls les consommateurs procèdent, en général, par paiement cash dans les transactions avec les détaillants.

Le règlement des transactions entre producteurs et collecteurs ou grossistes se fait, soit par crédit fournisseur octroyé avec un délai de règlement correspondant au temps d'écoulement du produit, soit par paiement cash.

Les transactions entre grossistes et détaillants, de même que celles qui se déroulent sur le marché de détail, sont soumises au libre jeu de la concurrence et sont réglées par paiement cash.

\section{STRUCTURE DU MARCHE}

La théorie économique considère que le marché de concurrence pure et parfaite, caractérisé par les fondamentaux suivants, est efficient : l'atomicité du marché ; la transparence et la fluidité de l'offre et la demande (libre circulation de l'information) ; la libre entrée et sortie de l'industrie et l'homogénéité du produit (impossible différenciation). A l'inverse, les marchés imparfaits (monopoles ; oligopoles ; concurrence monopolistiques, etc.) sont inefficients.

\section{Atomicité du marché}

Le marché est caractérisé par l'existence de plusieurs offreurs et demandeurs, dont aucun ne peut influencer significativement la formation des prix. De fait, le prix du marché reflète la disposition à payer des consommateurs et est fixé par le libre jeu de l'offre et la demande.

L'offre de riz local, (le riz non importé) est hétérogène du fait des nombreuses variétés existantes. On distingue le riz de plateau de celui de bas fond. On enregistre dans la zone de Gagnoa plusieurs variétés de riz local. Koné (2003) a donné les noms vernaculaires et les significations de certaines de ces variétés. Ce sont : riz de plateau- Papalémé, Sosso, le Nathalie (riz introduit par une dame du nom de Nathalie), Trin. Riz de bas-fond - Saka kpo (riz noir). Riz de plateau et de bas fond - Déadé, Sakigrédjo (dent pourrie), Zikpikli (grain court), Saka hiti (riz géant), et Saka kolotchê (petit grain long).

Au niveau des bas fonds, plusieurs variétés sélectionnées grâce à la recherche scientifique sont exploitées. II s'agit de WITA 9 et de Bouaké 189. Pour toutes ces variétés, la période d'abondance part de juillet à octobre et la période de pénurie de novembre à juin.

La demande de riz local provient d'une faible clientèle propre de sorte que les offreurs sont en situation de concurrence monopolistique sur ce marché marginal. Cependant, cette situation de quasi monopole n'affecte pas les prix. Mais, pour la majorité des consommateurs, le riz local et le riz importé sont parfaitement substituables. Ces consommateurs sont issus de toutes les catégories socio -professionnelles et de toutes origines.

\section{Transparence et fluidité du marché}

II n'existe certes pas de structure officielle de communication chargée d'informer les opérateurs de la filière de l'évolution de l'offre et la demande et des prix. Cependant, le «bouche à oreille» fonctionne parfaitement à travers les contacts privés et les réseaux informels de sorte que le prix pratiqué est très homogène. Sur le marché, l'offre s'adapte bien à la demande, ce qui se traduit par une grande variation des prix selon la rareté relative du produit.

\section{Libre entrée et sortie}

Les opérateurs entrent et sortent librement du marché puisque aucune législation n'exclue une certaine catégorie d'individus d'exercer dans la filière.

\section{L'homogénéité du produit}

Le riz local se différencie d'une manière générale, suivant quelques facteurs importants. Ce sont notamment, l'origine du produit, (riz de plateau ou de bas-fond), la variété, le type de 
transformation (étuvé ou non), le goût et les qualités culinaires. Cependant, pour une même variété, provenant d'une origine donnée, il n y a pas de différenciation du produit.

Au total, le marché du riz local est, malgré quelques imperfections, globalement concurrentiel.

\section{Variation du prix}

Le prix du riz local se caractérise par sa variation dans le temps (Tableau 4). Le prix de détail croît en moyenne de $20 \%$ en période de pénurie, passant de 257 à 309 F CFA, en moyenne par kilogramme. Par contre, la faiblesse des écarts types révèle une grande homogénéité du prix au cours des grandes périodes.

\section{Structure du prix de détail}

Le prix au consommateur du kilogramme de riz est décomposé en marges bénéficiaires et coûts supportés par chaque opérateur de la filière (Tableau 5).

\section{Détail des coûts}

Les coûts moyens de production de paddy s'élèvent à $54 \mathrm{~F} \mathrm{CFA/kg} \mathrm{en} \mathrm{milieu} \mathrm{urbain} \mathrm{contre}$ 65 F CFA / kg en milieu rural. Cette différence découle des frais de transports des intrants et de l'équipement qui en augmentent le prix de revient. Ces coûts sont composés essentiellement de coûts variables, $81 \%$ en milieu urbain et $82 \%$ en milieu rural
(Tableau 5). Les coûts moyens de commercialisation du grossiste s'élèvent à $13 \mathrm{~F} \mathrm{CFA} / \mathrm{kg}$ et $7 \mathrm{~F} \mathrm{CFA} / \mathrm{kg}$ pour le détaillant. Ces coûts sont dominés par les coûts variables ( $70 \%$ pour les grossistes et $60 \%$ pour les détaillants).

\section{Détail des marges}

La répartition des marges varie selon les périodes et les opérateurs (Tableau 5). Ainsi, dans le temps, les marges ont été plus élevées en période de pénurie. Au niveau des opérateurs, les marges des producteurs ont été le plus élevées quelque soit la période et la forme de vente.

\section{Comparaison des marges et des charges}

Globalement, le prix de détail est constitué majoritairement de marges bénéficiaires (57\% du prix en période d'abondance et $64 \%$ en période de pénurie). Les marges et les coûts sont également repartis entre les opérateurs. Ainsi, Les producteurs qui supportent $63 \%$ des coûts obtiennent $52 \%$ des marges en période d'abondance et $64 \%$ en période de pénurie. Les grossistes qui supportent $31 \%$ des coûts obtiennent $34 \%$ des marges en période d'abondance et $32 \%$ en période de pénurie. Les détaillants qui supportent $6 \%$ des coûts obtiennent $14 \%$ des marges en période d'abondance et $4 \%$ en période de pénurie.

Tableau 4 : Différents niveaux de prix dans la filière riz local.

Different levels of price in domestic rice channel.

\begin{tabular}{|c|c|c|c|c|}
\hline & \multicolumn{4}{|c|}{ Niveaux de prix (FCFA/kg) } \\
\hline & \multicolumn{2}{|c|}{$\begin{array}{l}\text { Période d'abondance } \\
\text { (juillet à octobre) }\end{array}$} & \multicolumn{2}{|c|}{$\begin{array}{c}\text { Période de pénurie } \\
\text { (novembre à juin) }\end{array}$} \\
\hline & Milieu rural & Péri urbain & Milieu rural & Péri urbain \\
\hline Prix bord champ paddy & $98(23)$ & $105(20)$ & $130(9)$ & $140(20)$ \\
\hline $\begin{array}{l}\text { Prix de vente en détail de riz blanc } \\
\text { par le producteur }\end{array}$ & & $221(40)$ & & $262(40)$ \\
\hline Prix de gros riz blanc & & $231(45)$ & & 293 (19) \\
\hline Prix de détail du riz décortiqué & $194(16)$ & $257(20)$ & $243(12)$ & $309(15)$ \\
\hline
\end{tabular}

Les chiffres entre parenthèses sont les écarts types 
Tableau 5 : Structure du prix de détail au consommateur du riz blanchi.

Retail price structure of rice to consumer.

\begin{tabular}{|c|c|c|c|c|}
\hline \multirow[b]{4}{*}{ Opérateurs } & \multicolumn{4}{|c|}{ Prix de détail (FCFA/kg) } \\
\hline & \multicolumn{2}{|c|}{$\begin{array}{l}\text { Période d'abondance } \\
\text { (juillet à octobre) }\end{array}$} & \multicolumn{2}{|c|}{$\begin{array}{l}\text { Période de pénurie } \\
\text { (novembre à juin) }\end{array}$} \\
\hline & Charges & Marge & Charges & Marge \\
\hline & \multicolumn{4}{|c|}{$(\%)$} \\
\hline $\begin{array}{l}\text { Producteur } \\
(1.4 \mathrm{~kg} \text { de paddy) }\end{array}$ & 29 & 28 & 25 & 39 \\
\hline Grossiste & & 18 & & 19 \\
\hline Décorticage & 10 & & 8 & \\
\hline Vente & 5 & & 4 & \\
\hline Détaillant & 3 & 7 & 2 & 3 \\
\hline Total filière & 47 & 53 & 39 & 61 \\
\hline $\begin{array}{l}\text { Prix de détail } \\
\text { (fcfa } / \mathrm{kg} \text { ) }\end{array}$ & \multicolumn{2}{|c|}{$257(20)$} & \multicolumn{2}{|c|}{$309(15)$} \\
\hline
\end{tabular}

${ }^{2}$ Pour obtenir $1 \mathrm{~kg}$ de riz blanc, il faut $1.4 \mathrm{~kg}$ de paddy produits avec $54 \mathrm{~F} \mathrm{CFA} / \mathrm{kg}$ plus $25 \mathrm{f}$ par $\mathrm{kg}$ de riz décortiqué, soit $101 \mathrm{~F} \mathrm{CFA} / \mathrm{kg}$

Le décorticage est très souvent manuel en milieu rural. Les frais financiers engagés, en l'occurrence l'amortissement du mortier et du pilon, sont négligeables, mais, la main d'œuvre féminine familiale utilisée a un coût d'opportunité.

Les charges commerciales comprennent la location des magasins, les coûts de main d'œuvre, les frais d'électricité, les frais de transport.

\section{DISCUSSION}

L'analyse de la structure de marché révèle que le riz local dispose d'une faible clientèle propre fidélisée, mais pour la grande majorité des consommateurs, le riz local et le riz importé sont parfaitement substituables. Le marché est globalement concurrentiel. Les opérateurs de la filière doivent donc rechercher l'efficience des systèmes de production et de commercialisation afin de produire et vendre à un prix compétitif. Les resultats sont en accord avec ceux de Dembele et al. (1987) qui soutiennent que le circuit de commercialisation du riz est caractérisé par une libre concurrence significative à tout niveau de la chaîne productiondistribution. Par contre, les observations faites par Kamara (1987) concernant le circuit des vivriers ne se sont pas confirmées pas. L'auteur a mentionné la faiblesse de la concurrence dans ce circuit du fait de la concentration d'une grande partie du marché entre les mains d'un nombre restreint de commerçants et l'entente entre ces derniers pour imposer leurs prix aux autres acteurs du marché.

Le rapport de la marge bénéficiaire au prix de vente indique que les producteurs retirent la marge la plus élevée, infirmant l'idée répandue selon laquelle dans le secteur agricole, les producteurs sont exploités par les intermédiaires commerciaux. Néanmoins, le ratio bénéfice-coût a été favorable, de sorte qu'on peut en déduire qu'ils bénéficient d'une juste rémunération de leurs efforts.

La longueur des cycles de commercialisation a été conforme à la norme observée Berger (1984) qui a noté que, généralement, le cycle de commercialisation du riz est long avec plusieurs intermédiaires commerciaux

Le rapport de la marge bénéficiaire au prix de vente, surtout pour les détaillants, est de $10 \%$ en période d'abondance et $12 \%$ en période de pénurie. II est nettement supérieur à la norme qui est située entre 5 et $8 \%$ pour le riz dans les pays en développement (N'dri, 2003). Poelmans (1997) a trouvé des résultats similaires entre détaillants et consommateurs de riz local et importé à Bouaké $(11 \%$ et $24 \%$ respectivement, à Korhogo. L'auteur a expliqué ces marges trop élevées par le manque de concurrence entre détaillants. A Gagnoa, cette raison nous a paru plausible. Les détaillants sont assez souvent isolés dans les quartiers, échappant ainsi à une véritable concurrence. De même, sur le marché (lieu physique), ils sont regroupés par petits nombres (souvent par affinité ethnique) et s'entendent tacitement pour déterminer des prix à pratiquer par tous.

L'étude révèle que les marges et charges sont également réparties entre les opérateurs, en accord avec l'idée selon laquelle les intermédiaires commerciaux exploitent les producteurs. Ce résultat confirme les travaux de Jones (1972) pour qui rien ne prouve que les 
longues chaînes d'intermédiaires accroissent les frais de commercialisation et seraient source d'inefficacité du marché.

De fait, certains commerçants, particulièrement les grossistes obtiennent des surprofits en mettant en œuvre des stratégies commerciales plus complexes impliquant des phénomènes spéculatifs et des stratégies de termaillage (achat et vente à terme).

La spéculation procède à l'achat et au stockage de paddy en période d'abondance puis à la vente de riz blanchi en période de pénurie. Cinquante cinq pour cent des commerçants affirment pratiquer cette stratégie, mais sur des quantités réduites, car le stockage suppose des conditions de conservation adéquates, et donc, des investissements.

Le termaillage est pratiqué par les intermédiaires commerciaux qui financent la production ou prêtent de l'argent aux producteurs devant faire face à des besoins pressants, notamment la scolarité des enfants et les frais médicaux. Aussi, ces derniers sont-ils contraints de leur vendre la production par contrat entre les deux parties.

La pratique des prêts aux paysans est à la défaveur de ces derniers, car ils vendent à un prix inférieur au prix du marché à terme. Diomande (1995) a observé ce même phénomène dans la région montagneuse de l'Ouest de la Côte d'Ivoire, où souvent, des producteurs vendent leur paddy à vil prix (jusqu'à $20 \mathrm{~F} \mathrm{CFA} / \mathrm{kg}$ ) afin de faire face aux frais de scolarisation de leurs enfants. L'affirmation de Dembele et al. (1987) selon laquelle le circuit de commercialisation du riz est caractérisé par une disponibilité à faire face, à tout moment, aux besoins financiers des paysans, doit être nuancée. De fait, le mode de paiement se révèle être un facteur déterminant du prix chez le paysan comme l'a noté Hirsch (1993).

L'extrême fluctuation des prix des produits vivriers dans le secteur traditionnel obsevée par Fraet (1995) se confirme dans la filière riz. Ces fluctuations sont essentiellement saisonnières.

L'étude infirme les remarques de Eicher et Baker (1984), selon lesquelles les systèmes de commercialisation de produits vivriers privés en Afrique subsaharienne souffrent d'un manque d'information dû au faible volume commercialisé, de l'instabilité de l'offre en raison des variations des conditions climatiques, des maladies et des conditions de l'entreposage et du manque d'homogénéité des produits.

\section{CONCLUSION}

Globalement, le marché du riz local est certes concurrentiel, mais on note des pratiques monopolistiques et oligopolistiques. Aussi estil impératif d'améliorer l'information économique sur le marché, afin de permettre plus de concurrence et donc une meilleure efficience des systèmes de commercialisation.

\section{REFERENCES}

Anonyme 1. 1997. Rapport d'activités du Ministère de l'agriculture et des ressources animales (MINAGRA). Abidjan, Côte d'Ivoire.

Anonyme 2. 1998. Recensement général de la population et de l'habitat (RGPH) de Côte d'Ivoire en 1998. Institut National de la Statistique (INS). Abidjan, Côte d'Ivoire.

Berger (M.) et (K.) Dezancey. 1984. briding the Gender Gap in Agriculture Extension ; Washington International Center for Research Women

Dahoun (S.). 1998. Caractérisation semi-détaillée des bassins versants de la zone agroclimatique de Gagnoa : contraintes socioéconomiques à l'adoption des techniques rizicoles. Mémoire de Maîtrise, Université de Bouaké, Côte d'lvoire. $60 \mathrm{p}$

Dembelé (N.), Dione (J.) et (J. M.) Staaz. 1987. Descripion et analyse de la structure du marché des céréales mil-sorgho-maïs ; Projet sécurité alimentaire, M.S.U, CESA, Bamako Mali

Diomandé (K.). 1995. Dévaluation et autosuffisance alimentaire : cas de la filière riz en Côte d'Ivoire. Rapport technique. Université de Cocody, Faculté des Sciences économiques et de Gestion, CIRES. Abidjan, Côte d'Ivoire.

Eicher (C. K.) et (D. C.) Baker. 1984. Etude critique de la recherche sur le développement agricole en Afrique Subsaharienne. CRDIMR 100 f. Ottawa. Canada. 421p

Fradet (P.). 1995. Compétitivité du riz ivoirien : impact de la dévaluation du franc CFA ; Université Pierre Mendes France. Grenoble 2. Faculté de Sciences économiques; DEA Economie internationale. $164 \mathrm{p}$. 
Goffin (R.). 1975. Analyse microéconomique. Mémentos Dalloz. Paris, France. 197p.

Henderson (J. M.) et (R. E.) Quandt. 1975. Microéconomique. Formulation mathématique élémentaire. Modules économiques. Dunod, Paris, France. 408p.

Hirsch (B.). 1993. Le riz et les politiques rizicoles en Côte d'Ivoire 1960-1993. Caisse française de développement, Département des politiques et des études. Paris, France.

Jones (W.). 1972. Marketing Saple food crops in tropical Africa ; Cornell University Press, Ithaca, New York, USA.

Kamara (M.). 1987 : Performance des circuits de commercialisation du riz et de la banane plantain au sud ouest de la Côte d'lvoire : Une analyse selon le genre ; CIRES, Abidjan, Côte d'Ivoire.

Koffi (C.), Koné (D.) et (W.) Yté. 2005. Pratiques paysannes en agriculture de bas fonds au centre ouest de la Côte d'Ivoire. In Récent développement de la production du riz en Afrique de l'Ouest. Agronomie Africaine. Numéro spécial (décembre 2005). Abidjan, Côte d'Ivoire. P 73 à 84
Koné (D.). 2003. Mise au point des techniques culturales et d'aménagement appropriés. In Systèmes de production en zone de basfonds. Bilan et perspectives. Revue interne des programmes de première génération du CNRA. 23 p. Abidjan, Côte d'Ivoire.

N'dri (A.). 2003. Etude des systèmes de commercialisation du riz local dans la zone de Gagnoa. DEA Université de Cocody. UFR Sciences économiques et gestion. Abidjan, Côte d'Ivoire. 54p.

Perrault (P.). 1984. Le cadre théorique de la commercialisation des produits agricoles en Côte d'Ivoire. Actes de séminaire biennal du CIRES, du 14 au 18 Mai 1984, Abidjan, Côte d'Ivoire. $20 \mathrm{p}$

Poelmans (A.). 1997. La commercialisation du riz en Côte d'Ivoire ; Document de travail $n^{\circ} 9$ bis IDESSA-K.U Leuven. Abidjan, Côte d'Ivoire.

Raju (V. T.) et (M.) Van Oppen. 1982. Marketing efficiency for selected crops in semi arid tropical indiana. ICRISAT, India $20 \mathrm{p}$

Yao (K.). 1985. Politique agricole. Le cas du riz en Côte d'Ivoire. Rapport technique. CIRES. Abidjan, Côte d'Ivoire. 25 p 